\title{
TECNOLOGÍAS PARA LA INCORPORACIÓN DE OBJETOS 3D EN LIBROS DE PAPEL Y LIBROS DIGITALES
}

\section{Technologies for incorporating 3D objects in paper and digital books}

\section{Carlos Carbonell-Carrera, José-Luis Saorín, Cecile Meier, Dámari Melián-Díaz y Jorge De-la-Torre-Cantero}
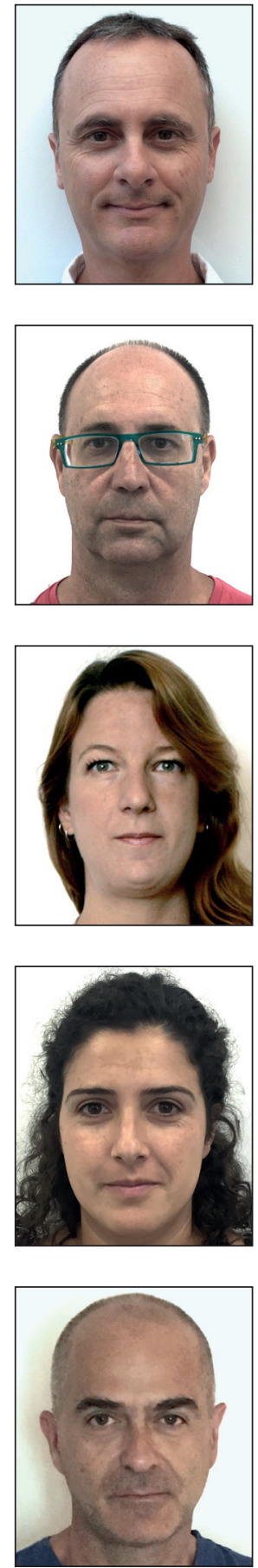

Carlos Carbonell-Carrera es profesor en la Universidad de La Laguna, e invited associate professor por la University of Applied Life Sciences and Environment de laşi, Rumanía. Es licenciado en documentación por la Universidad Oberta de Cataluña (2008), Master internacional en sistemas de información geográfica por la Universitat de Girona (2005) y doctor por la Universidad de La Laguna (2011).

http://orcid.org/0000-0003-4733-1598

ccarbone@ull.es

José-Luis Saorín imparte docencia en expresión gráfica en ingeniería y en innovación docente en el master de profesorado en la Universidad de La Laguna. Es doctor por la Universidad Politécnica de Valencia (2006) y técnico en diseño de programas, entornos y materiales con apoyo de las TIC por la Universidad Oberta de Cataluña. Es coordinador del grupo de investigación Dehaes de la Universidad de La Laguna, entre cuyas líneas de investigación se encuentra el diseño, creación y evaluación de objetos tridimensionales para educación.

http://orcid.org/0000-0003-3240-3317

jlsaorin@ull.es

Cecile Meier realiza su doctorado en la Facultad de Educación de la Universidad de la Laguna bajo el título: Incorporación del patrimonio escultórico en contextos educativos mediante el uso de impresoras 3D y tecnologías avanzadas de bajo coste. Es licenciada en bellas artes, especialidad de diseño gráfico, con un máster en diseño gráfico por la Universidad Politécnica de Valencia. Realizó un master en formación del profesorado, especialidad dibujo diseño y artes plásticas, por la Universidad de La Laguna (2012/2013).

http://orcid.org/0000-0001-7160-2154

alu0100305944@ull.edu.es

Dámari Melián-Díaz finalizó en 2011 sus estudios de arquitectura por la Universidad de Las Palmas, posteriormente realizó el máster en formación del profesorado en la especialidad de dibujo, diseño y artes gráficas. En la actualidad, cursa el tercer año de doctorado en educación por la Universidad de La Laguna, bajo el titulo Aplicación de tecnologías avanzadas de bajo coste como estrategia para la incorporación de competencias artísticas y creativas en los procesos de enseñanza-aprendizaje del dibujo técnico.

http://orcid.org/0000-0002-7651-728X

damarimd@gmail.com

Jorge De-la-Torre-Cantero es profesor asociado del Departamento de Técnicas y Proyectos en Ingeniería y Arquitectura de la Universidad de La Laguna desde 2003. Doctor en 2013 por la Universidad Politécnica de Valencia e investigador del Laboratorio de Fabricación Digital de la Universidad de La Laguna. Sus líneas de investigación son: STEAM (science, technology, engineering, arts, and mathematics: influencia del arte y el diseño en las disciplinas científico-tecnológicas); tecnologías gráficas avanzadas, fabricación digital y tecnologías BIM (building information modeling).

http://orcid.org/0000-0001-5516-0456

jcantero@ull.es 


\title{
Resumen
}

El libro, como elemento de transmisión de conocimiento, muestra la información mediante texto, imágenes y dibujos. Cuando el texto contiene información de carácter tridimensional o geométrico, para complementar el libro, se utilizan recursos complementarios como objetos tangibles, desplegables o maquetas que facilitan y complementan la interpretación del texto. Con la aparición de los libros digitales es posible incorporar formatos multimedia como vídeos, gráficos interactivos, galerías de fotos u objetos 3D. Este artículo describe las posibilidades de incluir modelos tridimensionales en libros en formato tradicional de papel, así como en libros en versión electrónica tanto para PC como para dispositivos móviles. Para este trabajo se han realizado varios prototipos de libros en varios formatos y se presentan instrucciones para que el lector pueda visualizar los objetos 3D incluidos en los mismos.

\section{Palabras clave}

Códigos QR; Libro digital; Modelos tridimensionales; 3D; Pop-up; Paper toy; Realidad aumentada.

\begin{abstract}
Traditionally, the book uses text, pictures, and drawings to show information. To complement the information, various methods have been used, such as adding tangible objects or models. With the advent of digital books, it is possible to incorporate videos, interactive graphics, photo galleries, and 3D objects. This article describes the possibilities of including 3D models in books that are paper or electronic (read on a desktop or mobile device). For this article we have made several book prototypes in various formats, and instructions are presented so that the reader can visualize the 3D objects contained in them.
\end{abstract}

\section{Keywords}

QR codes; Digital book; Three-dimensional models; 3D; Pop-up; Paper toy; Augmented reality.

Carbonell-Carrera, Carlos; Saorín, José-Luis; Meier, Cecile; Melián-Díaz, Dámari; De-la-Torre-Cantero, Jorge (2016).

"Tecnologías para la incorporación de objetos 3D en libros de papel y libros digitales". El profesional de la información, v. 25, n. 3 , pp. $661-670$.

http://dx.doi.org/10.3145/epi.2016.jul.16

\section{Introducción}

Los libros, en sentido amplio, han sido el formato por excelencia para la difusión de contenidos. El acceso a información digital en línea, a través de foros, sitios webs, documentos auto-editados para su descarga, etc. supera al que se realiza mediante el formato clásico de libro para la lectura. La función del libro tradicional, tanto de texto como divulgativo, sigue teniendo vigencia en pleno siglo XXI para la difusión de ideas y conocimiento. En educación universitaria los libros siguen siendo el recurso más frecuentemente recomendado (Rodríguez-Bravo et al., 2015).

Los libros, uno de los principales vehículos de transmisión de información, deberían poder incluir imágenes tridimensionales en su interior

Al tradicional formato de libro en papel se ha sumado el libro electrónico. Hay múltiples definiciones de libro electrónico: puede considerarse una versión digital de un libro, es decir, cualquier texto legible en pantalla o cualquier fichero en formato digital que puede descargarse en dispositivos electrónicos para su posterior visualización (Rao, 2005; Doctorow, 2004; Berube, 2005; Kang; Wang; Ling, 2009). El libro electrónico, sobre todo si es de texto, también puede ser entendido de forma más compleja, en el contexto de los objetos educativos digitales (Gértrudix-Barrio et al., 2007), puesto que sus posibilidades de contenido, difusión, lectura, reutilización, interrelación con otros contenidos y muchos otros aspectos pueden ser repensados de forma innovadora en el espacio digital.
En ámbitos educativos, los objetos y modelos en tres dimensiones son utilizados de manera habitual en:

\footnotetext{
- ingeniería y arquitectura (maquetas);

- geografía (mapas con relieve);

- dibujo técnico y las vistas normalizadas (piezas técnicas);

- estudios artísticos (réplicas de obras escultóricas);

- para conocer órganos en clases de anatomía, elementos naturales en biología, etc.
}

En la figura 1 se muestra un ejemplo de maqueta y lámina de estudio utilizados en enseñanza superior. En diversos estudios (Andrade-Lotero et al., 2012) se ha demostrado que un contenido se aprende más rápido mediante la manipulación de objetos tridimensionales.

Los libros, tanto electrónicos como de papel, al ser uno de los principales vehículos de transmisión de información, deberían disponer de la posibilidad de incluir objetos tridimensionales en su interior, ya que dichos objetos son diferentes a otros que pueden incluirse en un libro, como imágenes o videos. La interpretación detallada de la tridimensionalidad de un objeto a través de imágenes bidimensionales es un proceso que requiere, en algunos casos, un gran esfuerzo intelectual. Sin embargo, los modelos 3D, al permitir la observación desde distintos puntos de vista, facilita el aprendizaje de los conceptos espaciales asociados a los mismos.

En un escenario donde conviven los dos formatos: electrónico y papel, es interesante conocer de qué manera se puede conseguir que los objetos 3D se incluyan en los libros. En este artículo se describen y comparan nueve tecnologías para incorporar un objeto 3D con el que se pueda interactuar (mover y visualizar desde distintos ángulos) tanto en 

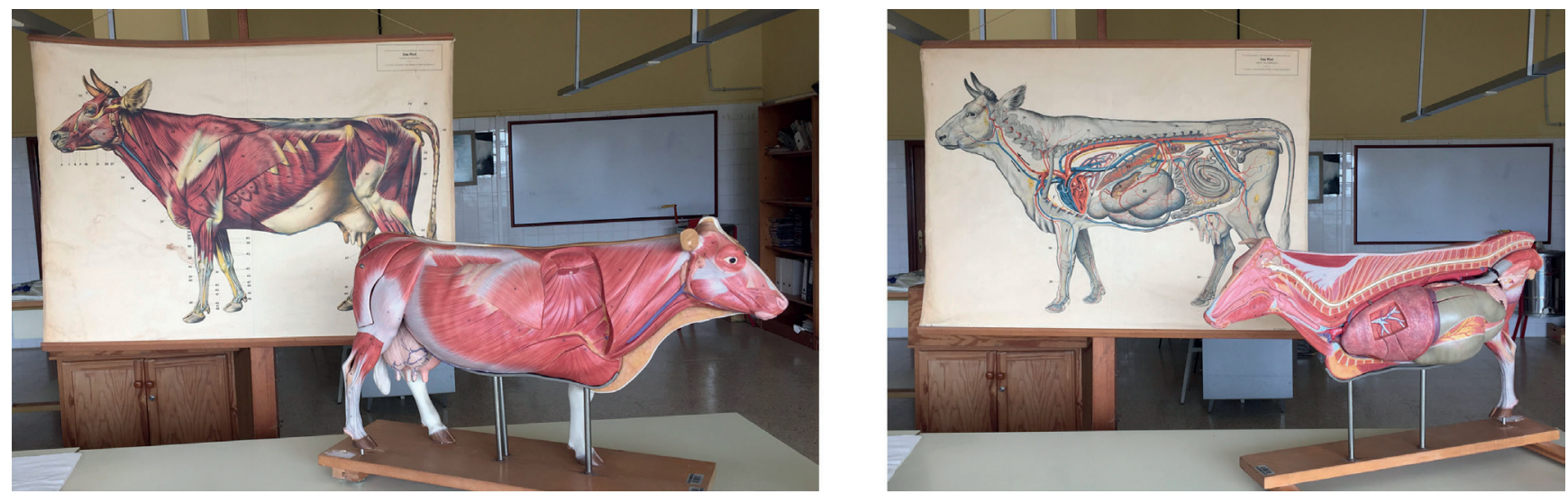

Figura 1. Lámina y maqueta de estudio (modelo de vaca de la empresa Medical Simulator). Área de Zootecnia de la Universidad de La Laguna

libros de papel como en libros electrónicos. Aunque la inclusión de objetos tridimensionales se orienta más hacia el sector del libro de texto o divulgativo en los campos del arte, naturaleza, ciencia o tecnología, por citar los más extendidos, las tecnologías presentadas en este artículo son válidas para cualquier tipo de texto.

\section{Incorporación de objetos 3D en libros}

El intento de incorporar la tercera dimensión en los libros de texto o documentos informativos no es nuevo, tuvo su aparición hace siglos. Tanto con los libros pop-up como mediante el uso de imágenes en perspectiva (Damisch, 1997) se lograba el efecto de mostrar esa tercera dimensión. Sin embargo, las imágenes en perspectiva siguen siendo bidimensionales y no permiten visualizar el objeto desde un punto de vista distinto al representado. Los visualizadores 3D digitales se basan en las técnicas de representación clásicas, por lo que en realidad vemos una imagen $2 \mathrm{D}$ en la pantalla del ordenador o dispositivo de visualización. Sin embargo, dicha imagen es redibujada instantáneamente cuando el usuario cambia el punto de vista con un controlador (un ratón, el dedo...), por lo que se produce la sensación de manipular un objeto tridimensional.

La aparición del libro digital y tecnologías como la realidad aumentada permiten crear nuevas formas de visualización tridimensional. Con tecnologías digitales accesibles (muchas de ellas gratuitas), es posible actualizar las técnicas tradicionales para la incorporación de objetos 3D en formatos de papel como pueden ser los libros con pop-ups o los recortables. En este artículo se presentan nueve tecnologías para de incorpora- ción de objetos 3D en libros (entendida como la posibilidad de que un objeto se pueda manipular o visualizar desde distintos ángulos), tanto en formato digital como en papel (figura 2).

Para la incorporación de un objeto 3D en un libro partiremos en todos los casos de su producción mediante tecnologías digitales, tanto si el destino es el soporte papel como si es digital. Para ilustrar cada una de las posibilidades descritas se ha seleccionado como ejemplo el modelo 3D digital de la escultura Monumento al gato del artista español Óscar Domínguez (figura 3), que formó parte de la I Exposición internacional de esculturas en la calle en Santa Cruz de Tenerife entre 1973 y 1974. El modelo 3D ha sido creado con la aplicación gratuita SketchUp.

\subsection{Incorporación de objetos 3D en libros de papel}

\subsubsection{Libros pop-up (a partir de software digital)}

En libros con pop-up, al abrir sus páginas se despliega un

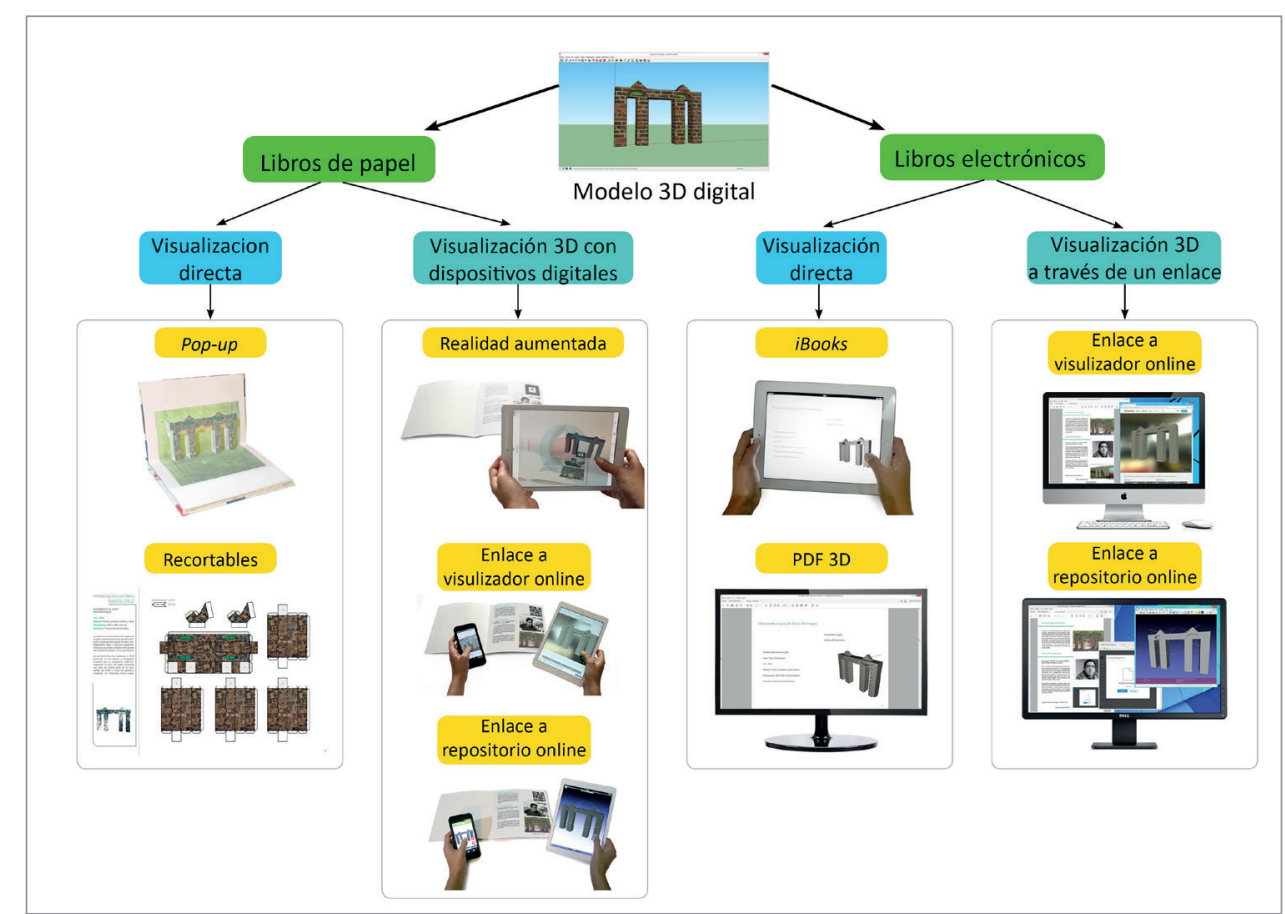

Figura 2. Tecnologías para la incorporación de objetos 3D en libros 


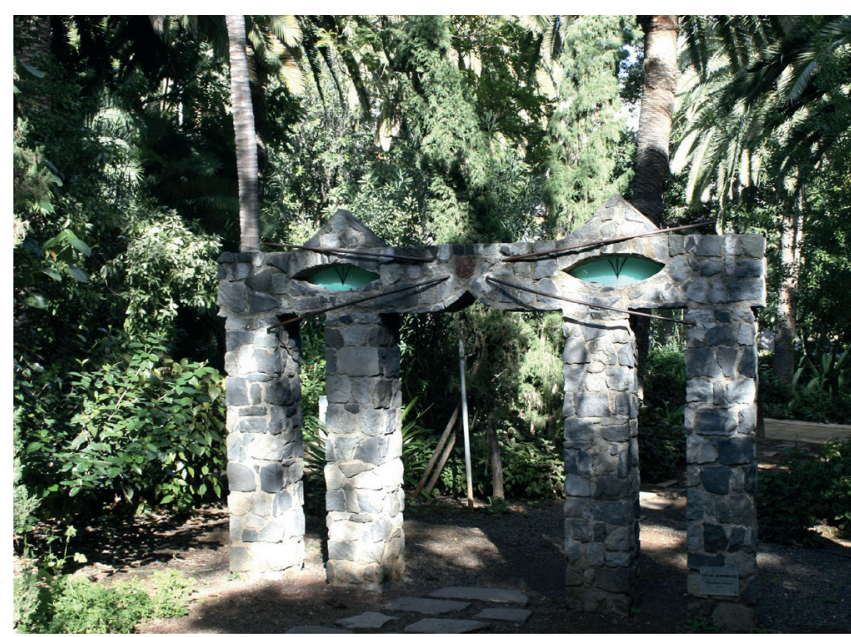

Figura 3. Monumento al gato de Óscar Domínguez

modelo tridimensional de papel que permite una mejor comprensión de los contenidos volumétricos desarrollados en el texto. Existen referencias de estos libros desde 1240, cuando apareció el primero datado hasta la fecha (ConnoIly, 1999). Han sido utilizados en el campo de la medicina y en ámbitos educativos (Glassner, 2002), y se siguen empleando en la actualidad, sobre todo en el ámbito infantil y libro-juego, como método para incorporar objetos 3D en los libros de papel (figura 4).

El conjunto de técnicas para el diseño y creación de libros con objetos 3D desplegables ( $p o p-u p$ ) de papel se denomina ingeniería de papel, término que hace referencia al conjunto de conocimientos para el diseño con papel, a través de sus posibilidades de plegado, recorte y ensamblado, dotándolos de tridimensionalidad.

Para el diseño de pop-ups es necesario entender el mecanismo de plegado y determinar las posiciones de los objetos emergentes para que no choquen entre sí o sobresalgan de la página (lizuka et al., 2011). Con aplicaciones como Pop-up Card Designer Pro (disponible en versión gratuita) o Popup Card Studio, es posible crear pop-ups de manera digital. Estos programas permiten dibujar el contorno de un objeto y crear las líneas de recortado y doblado para construir la tercera dimensión. En la figura 5 se muestra el pop-up del objeto 3D utilizado como ejemplo en este artículo. https://goo.gl/LYOk9X

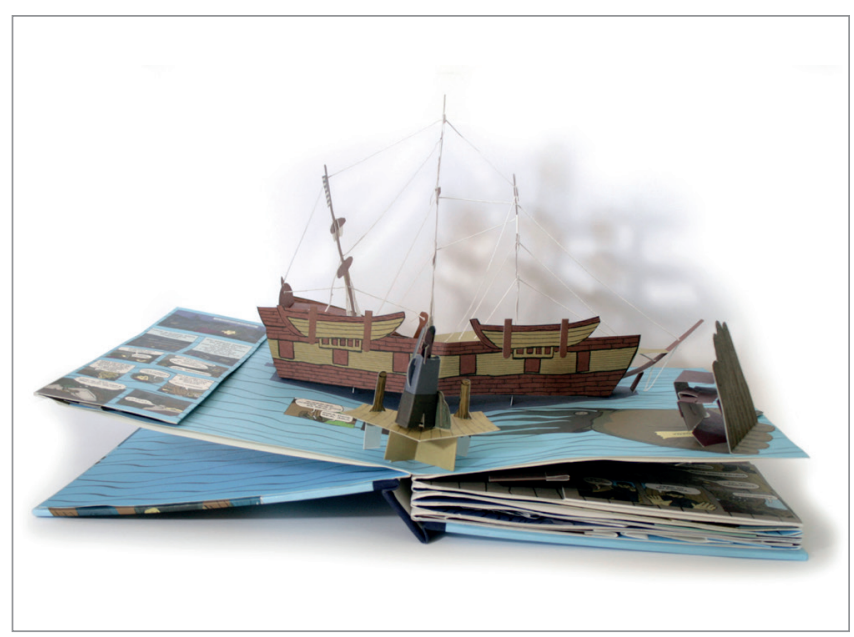

Figura 4. Libro pop-up

La ventaja del libro pop-up radica en que, una vez terminado el proceso de creación, permite acceder a los modelos 3D tan sólo con abrir el libro, sin necesidad de ninguna tecnología ni procedimiento adicional; es por ello que estos libros son utilizados con frecuencia en educación infantil y primaria, sobre todo como material complementario para actividades, biblioteca o consumo particular. Por otro lado, un libro pop-up no es fácilmente modificable y requiere de un proceso de fabricación costoso en tiempo, material y mano de obra.

\section{Se describen nueve tecnologías para in- corporar un objeto 3D con el que se pue- da interactuar tanto en libros de papel como en libros electrónicos}

\subsubsection{Libros con papertoys (recortables a partir de mode- los digitales 3D)}

En los libros con papertoys, el lector mediante recorte y plegado de papel puede obtener modelos tridimensionales a partir de patrones de piezas que se unen con pegamento. Los primeros recortables aparecen en Europa en el siglo XVIII, en el París de Luis XV, aunque es en 1840, con la aparición de la litografía en color, cuando experimentan
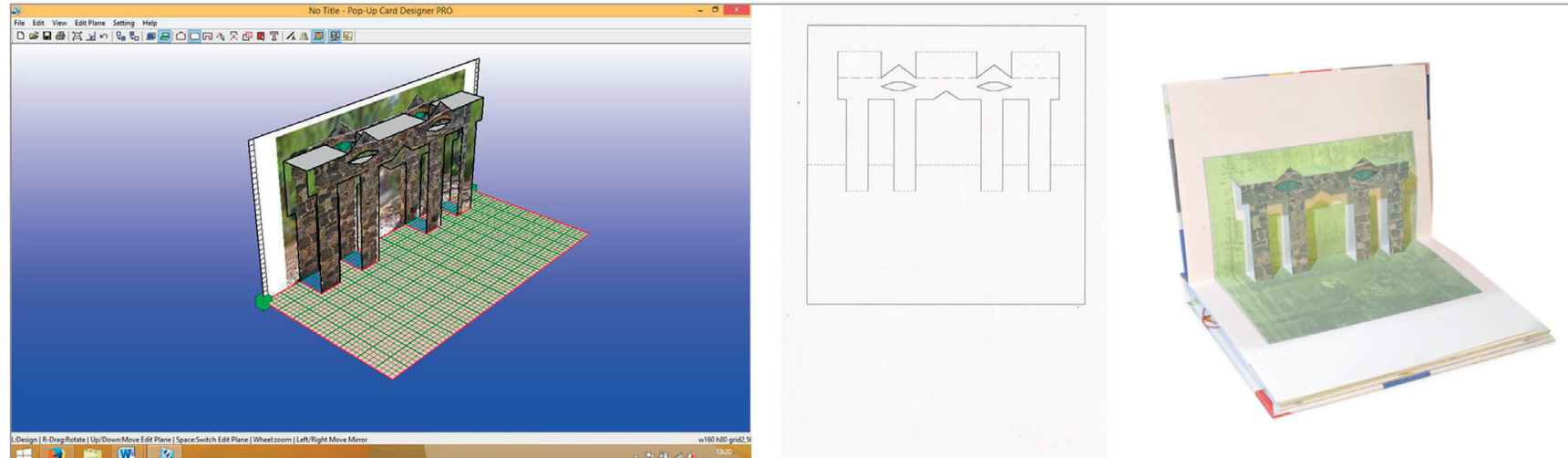

Figura 5. Ejemplo de un pop-up construido en Pop-up Card Designer Pro 

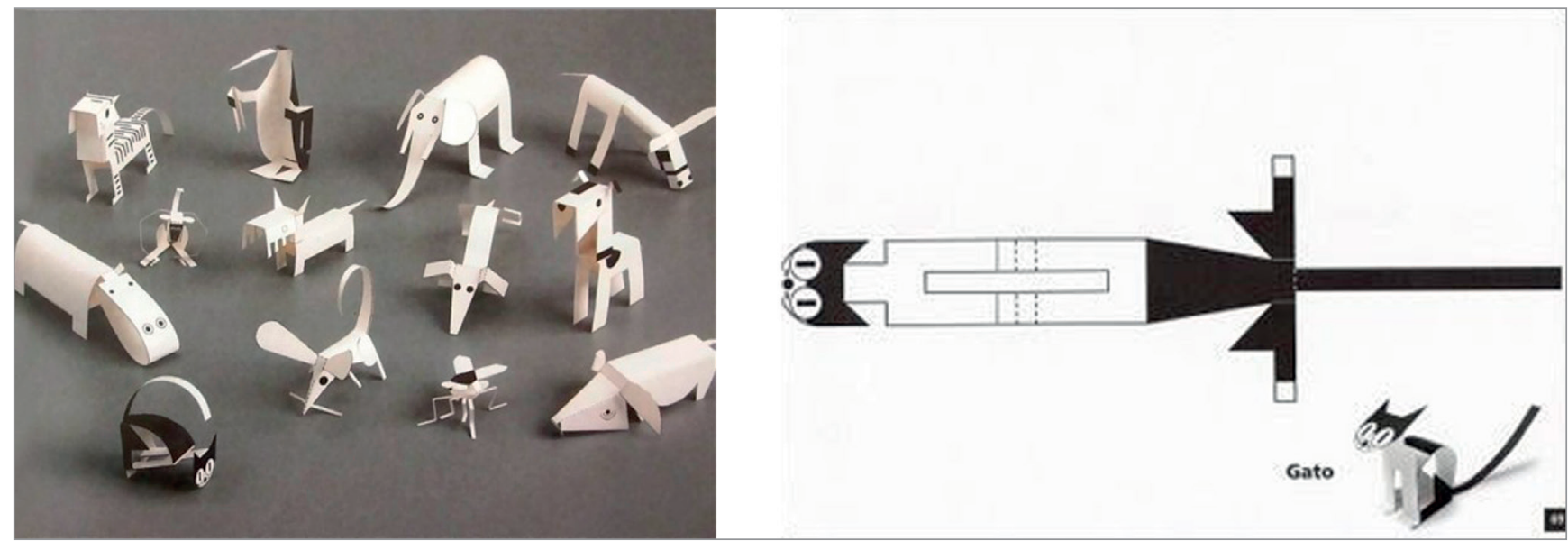

Figura 6. Ejemplo de libro de recortables

su mayor desarrollo y difusión (Haining, 1979; Reid-Walsh, 2012). Una muestra temprana de papertoys en España es la propuesta llevada a cabo por Antonio de Lara en los años 1933-1934 para la revista Crónica: se trata de una serie de recortables y plegables de animales coleccionables llamada El arca de Noé (IVAM, 2004) (figura 6).

\section{El formato PDF 3D, una vez resueltos los} problemas de visualización en dispositivos móviles, podría convertirse en el estándar para la inclusión de objetos 3D en libros digitales

El diseño de un papertoy con herramientas digitales constituye un proceso que engloba dos fases:

- la primera consiste en crear una plantilla recortable del modelo tridimensional, para lo cual se pueden utilizar aplicaciones como 123D Make, Pepakura Designer o extensiones de SketchUp como Unwrap and Flatten, Unfold o Flattery (figura 7);

- en la segunda fase se lleva a cabo un proceso de posproducción para añadir detalles como solapas, pestañas y texturas con programas como AutoCad Illustrator, InDesign o Gimp.

El diseño de un libro de recortables es una manera económica para la divulgación de objetos 3D en un aula sin acceso a internet ni dispositivos digitales. La creación de las planti-
Ilas recortables necesita varios programas, así como conocimientos informáticos y de retoque de imagen, pero el montaje final de los modelos 3D los realiza el lector con la única ayuda de unas tijeras y una barra de pegamento. Una vez realizados, son fácilmente reproducibles a través de fotocopias y posibles de incorporar en cualquier entorno escolar. El ejemplo del objeto 3D utilizado en este artículo (figura 8), además de otras 11 esculturas de Santa Cruz de Tenerife convertidas a formato pop-up se puede consultar en: https://goo.gl/kgybmY

\subsubsection{Libros de papel con objetos 3D en realidad aumen- tada (Magic book)}

La realidad aumentada (RA) permite al usuario ver el mundo real a través de un dispositivo digital con objetos virtuales superpuestos (Azuma, 1997). Los objetos virtuales pueden ser manipulados por el individuo, coordinando sus movimientos con las manos para obtener el punto de vista que desea. Se usa en distintos contextos: militar, medicina, diseño, ingeniería, robótica, enseñanza y aprendizaje, entretenimiento, tratamientos psicológicos, etc. (Azuma, 1997; Azuma et al., 2001).

La evolución de esta tecnología ha dado lugar a la aparición del libro aumentado o magic book (Billinghurst; Kato; Poupyrev, 2001; Juan; Beatrice; Cano, 2008; Ucelli et al., 2005). Este tipo de libro contiene unas marcas, las denominadas marcas de realidad aumentada, que permiten ver un objeto
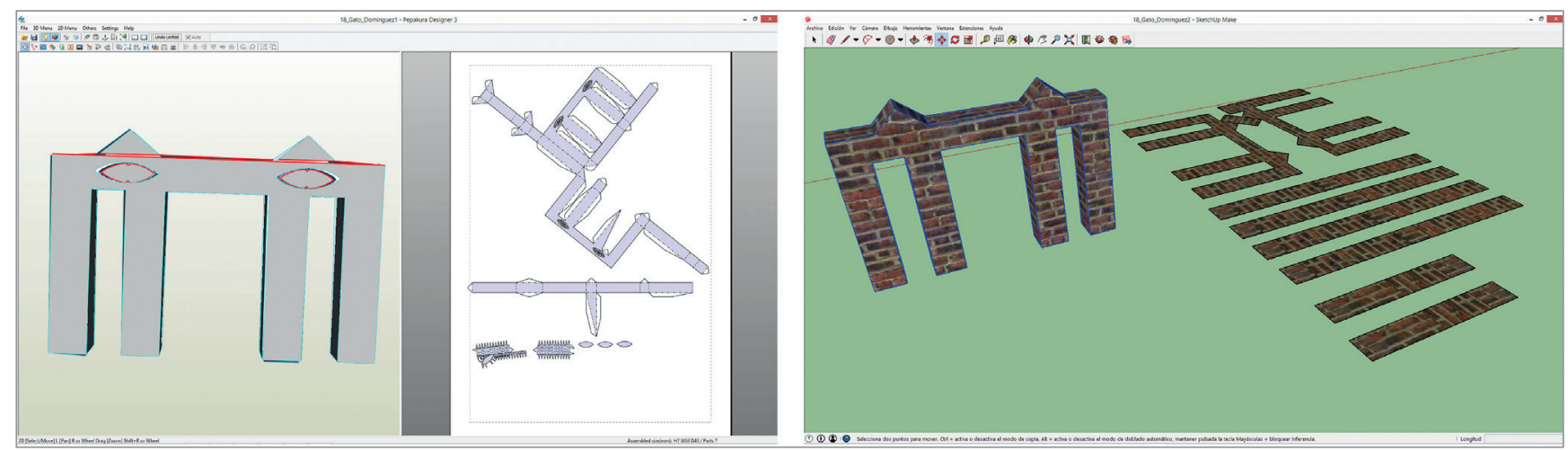

Figura 7. Ejemplos de creación de desplegado digital con Pepakura Designer (izq.) y Unwrap and Flatten de Sketchup Make (dcha.) 


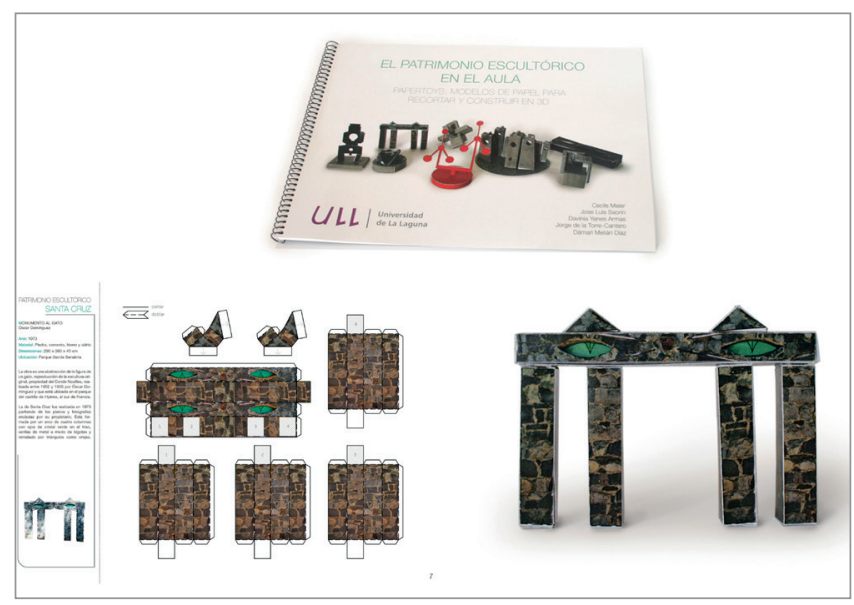

Figura 8. Libro de recortables con información (izq.) y el modelo montado en papel (dcha.)

3D a través de la cámara de un dispositivo móvil o la cámara de un ordenador (figura 9). Los libros aumentados ofrecen instrucciones del funcionamiento de la tecnología, donde se detalla la aplicación de visualización requerida para ver la realidad aumentada.

Un libro aumentado, es decir, un libro con marcas para visualizar realidad aumentada, permite convertir un modelo 3D en un archivo para visualizar a través de un dispositivo digital o una cámara de ordenador

Para crear un libro aumentado, es decir, un libro con dibujos o marcas para visualizar RA, existen programas, algunos con versiones gratuitas, como Augment, Layar, Aurasma, Augmentaty, ARMedia, etc. Estos programas permiten convertir un modelo $3 D$ en un archivo para visualizar en RA a través de un dispositivo digital o una cámara de ordenador, enfocando a una marca (figura 9).

Para este artículo se han creado los ficheros de RA con ARMedia de Inglobe Technologies, un plugin (complemento que añade una funcionalidad adicional a un software) gratuito en su versión educacional para el programa de modelado 3D SketchUp. Permite transformar un modelo tridimensional en un fichero de realidad aumentada. En la tabla 1 se detallan los pasos para la visualización en RA del ejemplo de este artículo.

En este caso, a diferencia del anterior, aunque el libro es de papel es necesario usar un dispositivo digital con una aplicación específica y el modelo descargado para visualizarlo. Es importante señalar que para realizar la descarga del visualizador y del fichero de RA se precisa disponer de conexión a internet, aunque una vez realizada dicha descarga, el sistema funciona sin conexión a la Red.

La RA permite interactuar con el modelo 3D, movernos alrededor del mismo, hacer zoom, y girarlo con gestos de nuestras manos. Sin embargo, este proceso es sensible a las

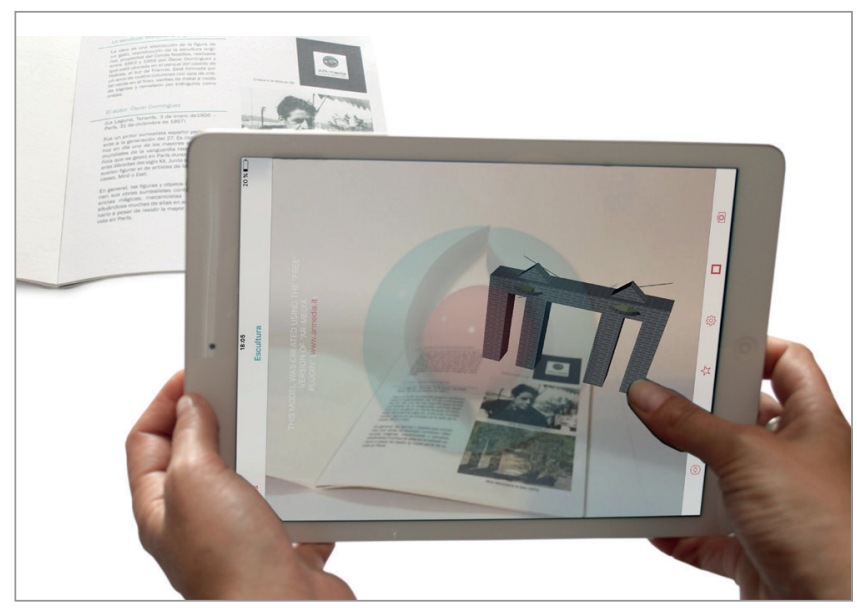

Figura 9. Visualización del modelo 3D mediante realidad aumentada

condiciones externas como puede ser la luz, la posición de la marca, o el tipo de movimiento requerido, ya que si la cámara deja de ver la marca, se deja de visualizar el objeto 3D.

\subsubsection{Libro de papel con enlaces a visualizadores 3D online}

Uno de los medios utilizados para conseguir que un libro de papel incluya información digital es mediante la referencia a una web, lo que obliga al lector a teclear la dirección web. Esta operación puede ser engorrosa ya que las direcciones suelen tener caracteres alfanuméricos complejos y de gran extensión. Existen estrategias para simplificar este proceso. Una de ellas es utilizar las direcciones url cortas y otra emplear aplicaciones que permiten, mediante un código, acceder a la dirección indicada, por ejemplo un código QR.

El código QR (Quick Response) fue creado en 1994 por la compañía japonesa Denso Wave. Los urls se convierten en una especie de código de barras que puede ser leído por las cámaras de los dispositivos móviles. Existen muchos generadores de código QR libres y gratuitos en internet (por ejemplo, $Q R$ code creator), que facilitan su generación y uso (Walsh, 2010).

http://www.qrstuff.com

Tabla 1. Instrucciones para la visualización en realidad aumentada (RA)

\section{Instrucciones para la visualización en RA del modelo 3D de la} escultura de Óscar Domínguez

Instalar la aplicación ARMedia Player de Inglobe Technologies en el dispositivo móvil digital (versión Android o IOS).

Descargar el fichero de RA "18_Gato_Dominguez.armedia" en el dispositivo digital.

https://goo.gl/bl23iG

Abrir con ARMedia Player

Seleccionar el modelo "18_Gato_Dominguez.armedia" (para ello es posible que haya que actualizar la base de datos de la aplicación)

Enfocar esta marca de RA con la cámara del dispositivo y visualizar el objeto en RA.

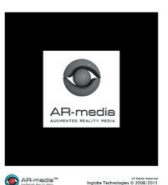




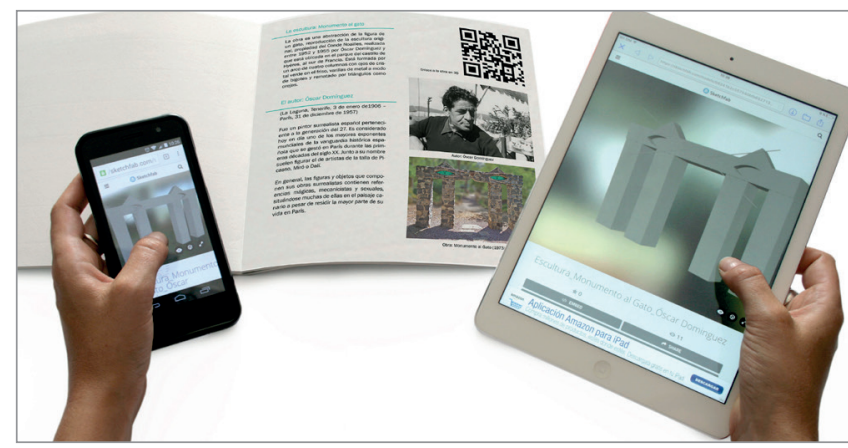

Figura 10. Visualización de un objeto 3D en SketchFab. Acceso desde un código QR. Tras cargar el modelo 3D en internet, es posible interactuar con el mismo girándolo y haciendo zoom directamente con los dedos. Una vez se ha visualizado el enlace ya no es necesario que el dispositivo siga enfocando al código QR (tabla 2).

Para incluir un modelo 3D en un libro de papel se puede usar un enlace o código QR que conecte con un modelo tridimensional que se visualiza directamente en el navegador de internet (figura 10). Existen webs especializadas en la difusión y/o venta de modelos tridimensionales (Thingiverse, Grabcad, 3D Warehouse, SketchFab, etc.). También algunos museos e instituciones están poniendo disponibles para visualizar online sus modelos 3D, como por ejemplo la Smithsonian Institution, o el proyecto para divulgación de patrimonio en 3D del Virtual World Heritage Laboratory:

http://3d.si.edu

http://vwhl.clas.virginia.edu

\section{El diseño de un libro de recortables es una manera económica de divulgar ob- jetos 3D en un aula sin acceso a internet ni dispositivos digitales}

Este sistema representa una alternativa más simple a la de visualización con realidad aumentada y además no requiere estar enfocando continuamente el código. Sin embargo sólo funciona mientras se disponga de conexión a internet. Además en muchos casos, debido a la configuración y políticas de los repositorios, no se permite descargar estos modelos en nuestros dispositivos. Esto no representa una gran limitación, siempre que nuestro objetivo sea la visualización del objeto 3D y no el disponer de él para por ejemplo editarlo o imprimirlo en 3D.

Tabla 2. Instrucciones para visualización online

Instrucciones para la visualización del ejemplo de este artículo desde un código QR enlazado a SketchFab

Instalar una aplicación de lector de código QR (por ejemplo QR code reader) en el dispositivo móvil

Abrir la aplicación y enfocar a la marca del código QR.

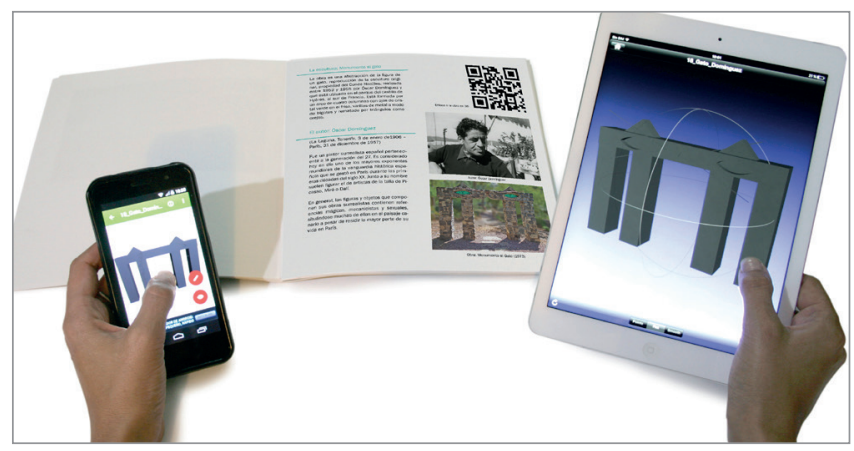

Figura 11. Modelo 3D en una aplicación de visualización de objetos 3D. Acceso desde un código QR. Al igual que con la RA, es posible interactuar con el modelo haciendo giros, cambios de escala y rotaciones directamente con movimientos de los dedos. Una vez está descargado el modelo 3D ya no es necesario que el dispositivo siga enfocando al código QR (tabla 3).

\subsubsection{Libro de papel con enlaces para descargar objetos tridimensionales}

Otra opción de incorporar objetos 3D en un libro es enlazar con repositorios de objetos 3D. Desde la aparición de aplicaciones como Dropbox, Drive, Mega o Box, que permiten almacenar información en la nube (internet), cualquier usuario puede cargar modelos 3D en la nube y compartir estos ficheros a través de un enlace, como puede ser un código QR.

Descargar, abrir o trabajar con texto, imágenes, gráficos o vídeos está normalizado en todos los dispositivos, sin embargo la visualización e interacción con modelos 3D digitales es poco habitual. Tradicionalmente sólo se podían visualizar archivos tridimensionales en programas de modelado 3D. En la actualidad esta limitación se ha superado, pudiéndose visualizar e interactuar con modelos tridimensionales en cualquier dispositivo digital que disponga de una aplicación de visualización 3D, como 3D Viewer, HD Model Viewer, A360, i3D Viewer, CAD View 3D, MeshLab, Graphite, etc. Una vez descargado y abierto el archivo, se queda en una galería dentro de la aplicación en nuestro dispositivo, permitiendo visualizarlo sin necesidad de conexión internet (figura 11).

Los libros de papel con enlaces a repositorios de objetos representan un proceso más complejo que el ejemplo ante-

Tabla 3. Instrucciones para visualizar objetos 3D en repositorios

Instrucciones para descargar y visualizar objetos 3D en repositorios

Instalar una aplicación de lectura de código QR (por ejemplo QR code reader) en el dispositivo digital.

Instalar el visualizador 3D, por ejemplo Meshlab (iOS) o Graphite (Android)

Enfocar la marca del código QR con la aplicación descargada.

Una vez en la carpeta del repositorio descargar el archivo con el objeto 3D seleccionado.

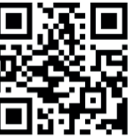

Utilizar el visualizador 3D para ver el objeto descargado. 


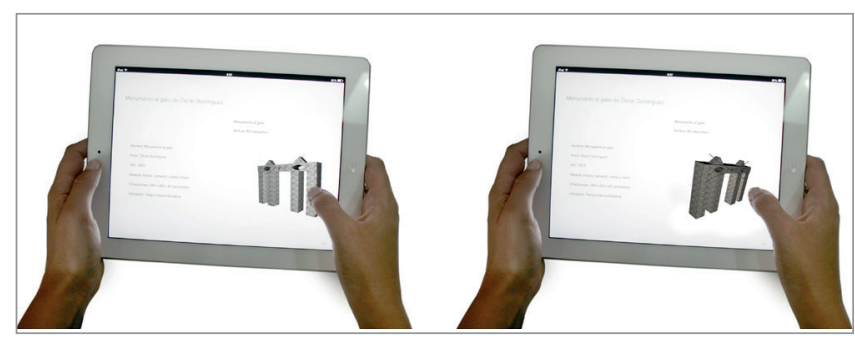

Figura 12. Visualización de un objeto 3D en un iBook

rior de enlazar a un visualizador 3D online, ya que implica (al igual que con la realidad aumentada) la instalación de una aplicación y la descarga de un fichero. Sin embargo, tiene la ventaja de que no requiere estar enfocando continuamente el código QR y que una vez descargados los objetos se pueden visualizar sin necesidad de conexión a internet ya que se quedan almacenadas en nuestro dispositivo.

\subsection{Incorporación de objetos tridimensionales en li- bros digitales}

Los libros digitales se pueden crear en múltiples formatos, no todos ellos con el mismo grado de estandarización (Browne, 2012). Prácticamente, cualquier formato de libro digital permite incluir con facilidad enlaces, imágenes, vídeo y sonido, una vez superados los problemas aún existentes de normalización en visores y tabletas. Sin embargo la mayoría de estos formatos no contempla incluir objetos tridimensionales, con excepción del formato PDF 3D o el iBook. A continuación se detallan cuatro opciones para incluir objetos 3D en libros digitales.

\subsubsection{Libro digital en formato iBook}

La aplicación iBooks Author presentada por Apple en 2012 permite incluir contenidos multimedia (imágenes, vídeos, cuestionarios, objetos 3D, animaciones, gráficos interactivos). En concreto, los modelos 3D hay que importarlos en formato Collada y una vez insertados pasan a formar parte integral del libro. Esta herramienta permite crear libros mul- timedia para dispositivos Apple, a través de un entorno gratuito de desarrollo muy intuitivo, pensado para profesores y divulgadores (Kwok, 2012).

La ventaja de un iBook es que una vez descargado el archivo, se puede visualizar el contenido 3D interactivo sin necesidad de conexión a internet, con lo cual reproduce a la perfección las características de movilidad de un libro de papel. Para este artículo, se ha realizado un iBook (figura 12) con modelos 3D que se pueden descargar y visualizar en cualquier dispositivo Apple (iPhone, iPad y Mac). https://goo.gl/sYdA95

\subsubsection{Libros digitales en formato Adobe PDF 3D}

El formato PDF ha sido durante años el estándar para documentos digitales imprimibles y, desde 2008, es la norma ISO 32000. El formato especial 3D-PDF permite incorporar contenido multimedia y modelos 3D (Ruthensteiner; Heß, 2008).

Para crear un PDF 3D se puede usar el programa Adobe PDF Pro, que permite diseñar un libro e incorporar contenido 3D. Sin embargo no es un programa diseñado para usuarios sin experiencia y además sólo está disponible de manera gratuita en versión de prueba de 30 días. EI PDF-3D se puede abrir con cualquier lector de ficheros PDF, sin embargo para la correcta visualización de los modelos 3D es necesario utilizar Adobe Reader. Este programa visualiza el fichero en cualquier dispositivo, aunque todavía presenta problemas en smartphones y tabletas digitales. Debido a ello se han creado otras aplicaciones, como 3D PDF Reader, que intentan mejorar la visualización en dispositivos móviles. Para este artículo se ha creado un ejemplo (figura 13) de PDF-3D que se puede descargar en: https://goo.gl/Kxq5Co

El formato PDF es un formato ya estandarizado para texto e imágenes. Si se resuelven los problemas de visualización en dispositivos móviles, el PDF 3D, podría convertirse en el formato estándar para incluir objetos tridimensionales en libros digitales.
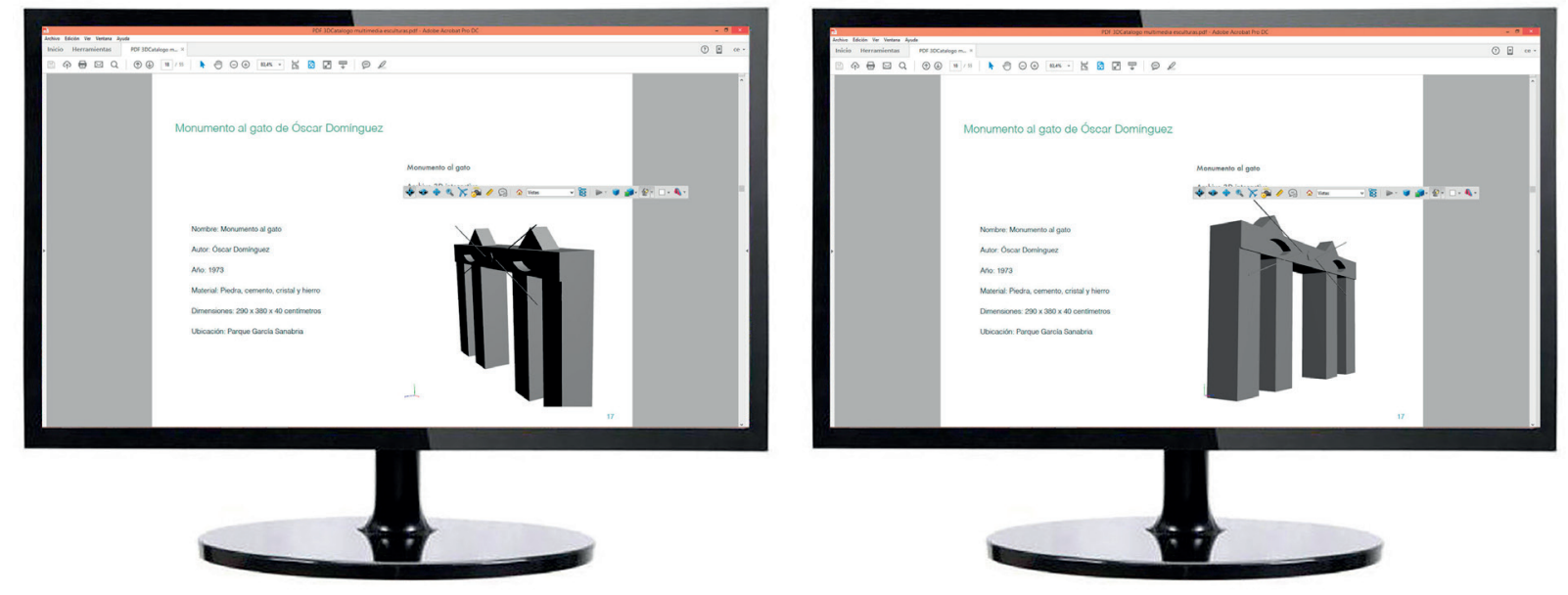

Figura 13. Visualización de un objeto 3D en PDF (Adobe Acrobat) 
2.2.3. Libro digital con enlaces a visualizadores 3D online y repositorios de objetos 3D

Incluir enlaces en un libro digital es una opción en todos los formatos existentes (PDF, epub, Word...). Los enlaces pueden ser a visualizadores 3D online o a repositorios de objetos 3D. Estas dos opciones son iguales que las detalladas en el caso de los libros de papel en los apartados 2.1.4. y 2.1.5. En este caso se ha realizado un ejemplo en PDF con enlace a la escultura del Monumento al gato de Óscar Domínguez que se puede descargar en:

https://goo.gl/BymCxz

Dicho ejemplo incluye dos enlaces, uno a un visualizador online y otro a un repositorio que permite la descarga del objeto 3D.

De esta manera cualquier persona puede crearse un documento digital que incluya objetos 3D en su contenido. Las limitaciones de cada uno de estos dos sistemas (descarga de aplicaciones y ficheros, conexión a internet, etc.) serán las mismas que las vistas en incluir objetos 3D a través de enlaces en los libros de papel.

\section{Conclusiones}

En este artículo se han descrito 9 propuestas para la inclusión de modelos tridimensionales en libros, tanto de papel como digitales. No todas las propuestas presentan las mismas características, por lo que se podrá optar por una u otra alternativa dependiendo de:

- tipo de libro que se quiera crear;

- recursos disponibles;

- conocimientos tecnológicos de los autores;

- destinatarios.

Por ejemplo, si los destinarios son estudiantes de educación infantil o primaria, lo normal, ya que no requiere ninguna formación, será utilizar libros de papel tipo pop-up o visualización directa en libros electrónicos como iBooks o PDF 3D. Los estudiantes en educación infantil o primaria no siempre tienen dispositivos móviles propios por lo que la utilización de libros electrónicos con contenido 3D directo, tendría que contar con materiales del centro escolar o de la propia familia del estudiante.

Para educación secundaria y superior, los libros (tanto de papel como digitales) con enlaces a visualizadores directos en internet (tipo SkechFab), pueden ser muy interesantes al permitir que los alumnos utilicen sus propios dispositivos móviles para ver los modelos 3D. Por otro lado, los libros con papertoys pueden ser una buena herramienta de divulgación mediante actividades de construcción de un objeto tridimensional en niveles educativos preuniversitarios.

Para autores que quieran incluir contenido 3D en sus libros, sería necesario un mínimo de formación tecnológica para poder crear o al menos manejar modelos 3D digitales. La manera más simple de incluir estos contenidos tridimensionales sería a través de enlaces (códigos QR o hipervínculos) a modelos ya creados en internet (ej. SketchFab). Autores con conocimientos avanzados pueden crearse sus propios papertoys, libros pop-up o repositorios de modelos 3D o realidad aumentada de una manera gratuita.
En ámbitos educativos, donde las aulas virtuales tienen una gran implantación, los libros digitales con contenidos tridimensionales son adecuados, por su formato, en este tipo de entornos de enseñanza-aprendizaje. Por otro lado, los contenedores online de objetos 3D, entendidos como pequeñas unidades de contenido, cada vez serán más habituales, pudiendo estar enlazados o no a libros digitales.

\section{Bibliografía}

Andrade-Lotero, Luis-Alejandro; Espitia-Gómez, Carolina; Huertas-Franco, Elquin-Antonio; Aldana-Ahumada, DerlyRocío; Bacca-Pachón, Paola-Andrea (2012). "Tocar o mirar: comparación de procesos cognitivos en el aprendizaje con o sin manipulación física". Psicología educativa, v. 18, n. 1, pp. 29-40.

http://dx.doi.org/10.5093/ed2012a3

Avella, Natalie (2003). Paper engineering: 3D design techniques for a 2D material. Rotovision. ISBN: 9782880467111

Azuma, Ronald (1997). "A survey of augmented reality". Presence, v. 6, n. 4, pp. 355-385.

http://www.cs.unc.edu/ azuma/ARpresence.pdf http://dx.doi.org/10.1162/pres.1997.6.4.355

Azuma, Ronald; Baillot, Yohan; Behringer, Reinhold; Feiner, Steven (2001). "Recent advances in augmented reality". IEEE Computer graphics and applications, v. 21, pp. 34-37. http://dx.doi.org/10.1109/38.963459

Berube, Linda (2005). "E-books in public libraries: a terminal or termination technology?". Interlending and document supply, v. 33, n. 1, pp. 14-18.

http://dx.doi.org/10.1108/02641610510582090

Billinghurst, Mark; Kato, Hirokazu; Poupyrev, Ivan (2001). "The magic book-moving seamlessly between reality and virtuality". IEEE computer graphics and applications, v. 21, n. 3, pp. 6-8

http://dx.doi.org/10.1109/38.920621

Browne, Glenda (2012). "Indexes in tomorrow's world". En: Australian Society for Technical Communicators (NSW) conference, Sydney.

http://www.webindexing.com.au/indexes-in-tomorrowsworld-astc-nsw-conference-presentation-november-2012

Connolly, Daniel K. (1999). "Imagined pilgrimage in the itinerary maps of Matthew Paris". The art bulletin, v. 81, n. 4, pp. 598-622.

http://www.jstor.org/stable/3051336?seq=1\#page_scan_ tab_contents

Damisch, Hubert (1997). El origen de la perspectiva. España: Alianza Editorial. ISBN: 8420671436

Doctorow, Cory (2004). Ebooks: Neither E, nor books. FQ Books.

http://craphound.com/ebooksneitherenorbooks.txt http://jamillan.com/doctorow.htm

Gértrudix-Barrio, Manuel; Álvarez-García, Sergio; Galistodel-Valle, Antonio; Gálvez-de-la-Cuesta, María-del-Carmen; Gértrudix-Barrio, Felipe (2007). "Acciones de diseño y desarrollo de objetos educativos digitales: programas ins- 
titucionales". Revista de universidad y sociedad del conocimiento (RUSC), v. 4, n. 1.

http://www.uoc.edu/rusc/4/1/dt/esp/gertrudix_alvarez_ galisteo_galvez.pdf

Glassner, Andrew (2002). "Interactive pop-up card design". Computer graphics and applications, v. 22, n. 1, pp. 79-86. http://dx.doi.org/10.1109/38.988749

Haining, Peter (1979). Movable books: An illustrated history. London: New English Library. ISBN: 9780450039492

lizuka, Satoshi; Endo, Yuki; Mitani, Jun; Kanamori, Yoshihiro; Fukui, Yukio (2011). "An interactive design system for pop-up cards with a physical simulation". The visual computer, v. 27, n. 6-8, pp. 605-612.

http://www.npal.cs.tsukuba.ac.jp/ iizuka/projects/popup/ data/fulltext.pdf

http://dx.doi.org/10.1007/s00371-011-0564-0

IVAM (2004). Los talleres didácticos del IVAM 1998-2005. Valencia: Instituto Valenciano de Arte Moderno. ISBN: 978 8448238605

Juan, Carmen; Beatrice, Francesca; Cano, Juan (2008). “An augmented reality system for learning the interior of the human body". En: Procs of the $8^{\text {th }}$ IEEE int conf on advanced learning technologies, pp. 186-188.

http://users.dsic.upv.es/ mcarmen/docs/HumanBody.pdf http://dx.doi.org/10.1109/ICALT.2008.121

Kang, Yen-Yu; Wang, Mao-Jiun; Ling, Rungtai (2009). “Usability evaluation of e-books". Displays, v. 30, pp. 49-52. http://dx.doi.org/10.1016/j.displa.2008.12.002

Kwok, Roberta (2012). "Going digital". Nature, v. 485, pp. 405-407.

http://dx.doi.org/10.1038/nj73

Rao, Siriginidi-Subba (2005). "Electronic books: their inte- gration into library and information center". The electronic library, v. 23, n. 1, pp. 116-140.

http://dx.doi.org/10.1108/02640470510582790

Reid-Walsh, Jacqueline (2012). “Books or toys?: A traveller's tale: Researching early movable books for and by children in material and virtual collections". Explorations into children's literature, v. 22, n. 1, pp. 156-169.

Rodríguez-Bravo, Blanca; Pacios, Ana-Reyes; Vianello-Osti, Marina; Moro-Cabero, Manuela; De-la-Mano-González, Marta (2015). "Digital transition of teaching learning resources at Spanish universities". El profesional de la Información, v. 24, n. 6, pp. 737-748.

http://dx.doi.org/10.3145/epi.2015.nov.05

Ruthensteiner, Bernhard; Heß, Martin (2008). “Embedding 3D models of biological specimens in PDF publications". Microscopy research and technique, v. 71, n. 11, pp. 778-786. http://dx.doi.org/10.1002/jemt.20618

Smithsonian Institution (2015). Smithsonian x3D. http://3d.si.edu/browser

Ucelli, Giuliana; Conti, Giuseppe; De-Amicis, Raffaele; Servidio, Rocco (2005). "Learning using augmented reality technology: Multiple means of interaction for teaching children the theory of colours". En: Procs of the $1^{\text {st }}$ intl conf on intelligent technologies for interactive entertainment, pp. 193-202. http://dx.doi.org/10.1007/11590323_20

Virtual World Heritage Laboratory (2015). The digital sculpture project.

http://www.digitalsculpture.org/laocoon/index.html

Walsh, Andrew (2010). "QR codes - Using mobile phones to deliver library instruction and help at the point of need". Journal of information literacy, v. 4 n. 1, pp. 55-65.

http://dx.doi.org/10.11645/4.1.1458

\section{Da visibilidad a tu trabajo depositándolo en e-LIS, el mayor repositorio internacional sobre biblioteconomía, documentación y comunicación}

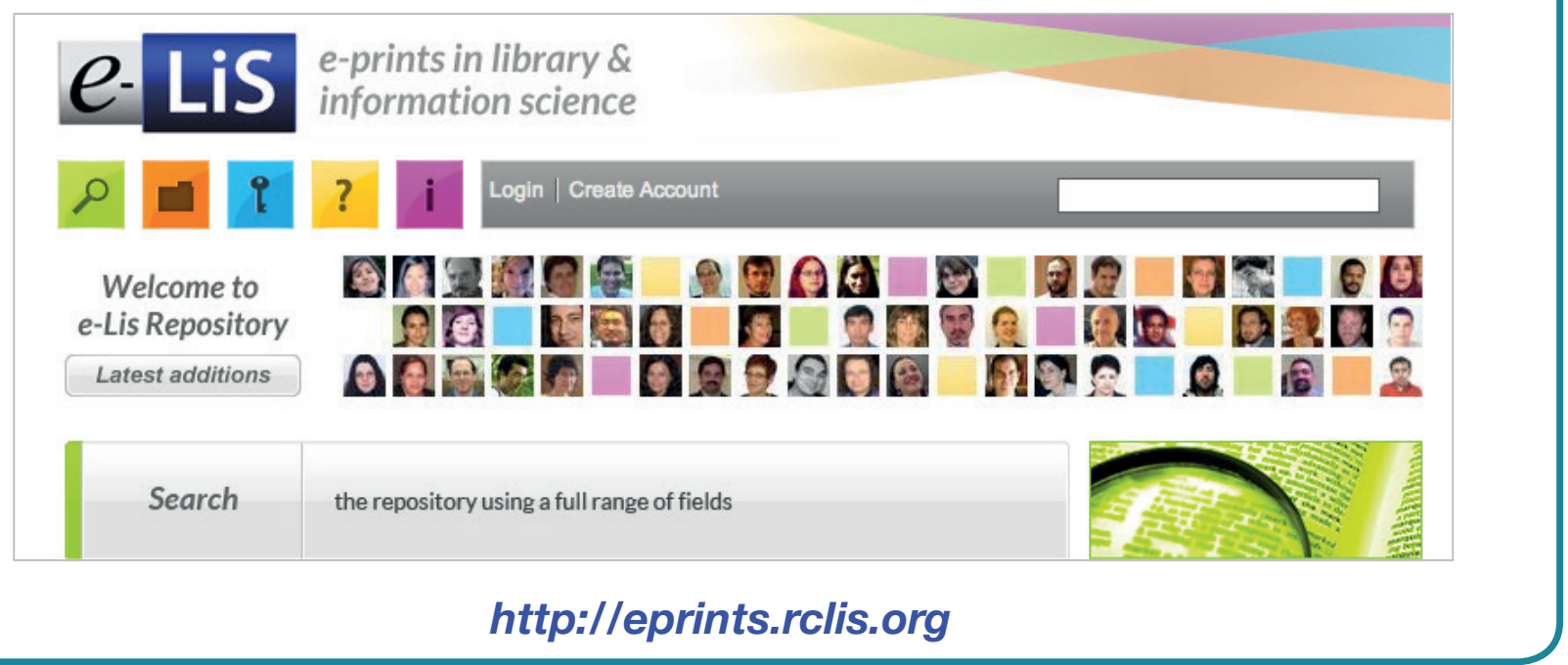

